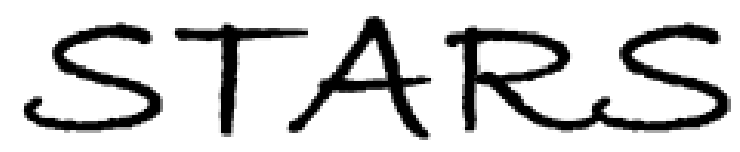

University of Central Florida

STARS

Faculty Bibliography 1990s

Faculty Bibliography

$1-1-1998$

\title{
Soret feedback in thermal diffusion of suspensions
}

Nelson V. Tabiryan

University of Central Florida

Weili Luo

University of Central Florida

Find similar works at: https://stars.library.ucf.edu/facultybib1990

University of Central Florida Libraries http://library.ucf.edu

This Article is brought to you for free and open access by the Faculty Bibliography at STARS. It has been accepted for inclusion in Faculty Bibliography 1990s by an authorized administrator of STARS. For more information, please contactSTARS@ucf.edu.

\section{Recommended Citation}

Tabiryan, Nelson V. and Luo, Weili, "Soret feedback in thermal diffusion of suspensions" (1998). Faculty Bibliography 1990s. 2467.

https://stars.library.ucf.edu/facultybib1990/2467

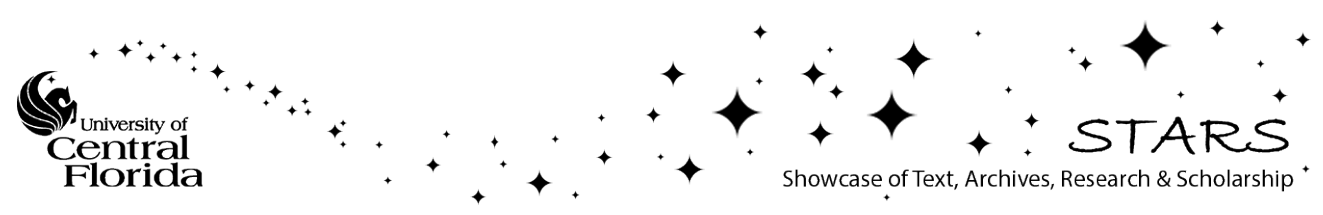




\title{
Soret feedback in thermal diffusion of suspensions
}

\author{
Nelson V. Tabiryan ${ }^{1, *}$ and Weili Luo ${ }^{2 \dagger}$ \\ ${ }^{1}$ Center for Research and Education in Optics and Lasers, University of Central Florida, P.O. Box 162700, \\ Orlando, Florida 32816-2700 \\ ${ }^{2}$ Physics Department, University of Central Florida, Orlando, Florida 32816 \\ (Received 28 January 1997; revised manuscript received 17 November 1997)
}

\begin{abstract}
We have theoretically studied the interaction of a light beam with liquids containing absorbing nanoparticles. We have shown that the Soret effect ensures a feedback that essentially limits heat insertion into the system and the change in its temperature: the temperature rise on the axis of a Gaussian beam is inversely proportional to the Soret constant. Transverse spatial redistribution of the absorbing particles gives a specific thickness dependence of the transparency of the material. These properties not only play an essential role in the study of light interactions with absorbing solutions, but also can underlie optical methods for measurements of the Soret constant. [S1063-651X(98)06704-X]

PACS number(s): $83.70 . \mathrm{Hq}, 66.10 . \mathrm{Cb}, 42.70 . \mathrm{Nq}$
\end{abstract}

\section{INTRODUCTION}

Colloidal suspensions have found a variety of important applications in modern technologies. Thus magnetic fluids are being largely used for polishing optical components $[1,2]$. Suspensions of silica particles in liquid crystals have exhibited extraordinary capabilities for optical storage [3,4]. Artificial media with high optical nonlinearity were obtained in colloids of submicrometer-size particles in liquids [5-7]. However, the physical mechanisms associated with many phenomena in colloids are still poorly understood, and optics serves as one of the most powerful methods for the study of such complex systems.

In the present paper, we study thermal diffusion phenomena that take place in solutions of absorbing centers like nanometer-size magnetic particles in kerosene, and dye molecules in liquid crystals. These experimental situations were intensely studied recently; see Refs. [8-12] for magnetic systems, and Refs. [13-15] for dye-doped liquid crystals. Our results may help to understand some of these interesting observations.

Concentration variations due to laser-beam-induced thermal diffusion in liquid mixtures (known as the Soret effect) were observed in a number of papers [16-20], and are recognized as one of the major mechanisms contributing to the interaction of light with liquid mixtures [21,22]. In the present paper, we show that a linear description of the thermal interaction of laser beams with complex media is not adequate in many cases, and may lead to nonphysical conclusions about changes on the temperature of the colloid containing absorbing particles. We reveal a mechanism of a transverse feedback induced by the Soret effect that limits the temperature increase in liquids containing absorbing particles and molecules. We show that a measurement of this temperature with the aid of a characteristic diffraction pattern

*Fax: (407) 823-6880.

Electronic address: nelson@ mail.creol.ucf.edu

†Fax: (407) 823-5112.

Electronic address: luo@pegasus.cc.ucf.edu may allow a straightforward determination of the Soret constant. A transverse modulation of the absorption constant and the refractive index due to the redistribution of the concentration of the particles and temperature leads to a nonlinear absorption with a specific thickness dependence of the absorbed power, and yields characteristic self-phase modulation patterns in the far field zone of the laser beam. We will find the spatial distribution of particles for a specific spatial profile of temperature obtained in light beams with a Gaussian profile of intensity.

\section{GENERAL EQUATIONS}

The equation of diffusion with account of the thermal diffusion effect can be written down in different forms [2325]. We will proceed from the equation

$$
\frac{\partial c}{\partial t}-D \Delta c=D_{T} \operatorname{div}[c(1-c) \operatorname{grad} T]
$$

neglecting hydrodynamic motions. In Eq. (1), $D$ is the mass diffusion constant (measured in $\mathrm{cm}^{2} / \mathrm{s}$ ); the parameter $D_{T}$ (measured in $\mathrm{cm}^{2} / \mathrm{s} \mathrm{K}$ ) is the coefficient of the thermal diffusion; and $c$ denotes the concentration of absorbing particles (or molecules) in the medium, and is defined as the ratio of the number of inclusions per unit volume to the total density of particles.

The constant of the thermal diffusion $D_{T}$ is a complex function of molecular parameters of individual species, their mass and size, and interaction forces. Even for a particular mixture, it is a complex function of temperature and density, and there are no universal laws found to describe these features for liquids. In our present discussion, we will assume $D_{T}$ to be constant in the range of temperature changes induced by the light beams. Generally speaking, this may not be correct, and the temperature and concentration dependence of the diffusion coefficients may affect quantitatively the effects we are going to discuss.

Equation (1) has to be complemented by the temperature conductivity equation 


$$
\frac{\partial T}{\partial t}-\chi \Delta T=\frac{\sigma I}{\rho C_{p}}
$$

where $\chi\left(\mathrm{cm}^{2} / \mathrm{s}\right)$ is the temperature conductivity coefficient, $\sigma\left(\mathrm{cm}^{-1}\right)$ is the absorption constant, $I\left(\mathrm{~W} / \mathrm{cm}^{2}\right)$ is the intensity of the laser beam, and $\rho C_{p}\left(\mathrm{~J} / \mathrm{cm}^{3} \mathrm{~K}\right)$ is the specific heat capacitance of the material.

In cases where variation of $c$ in space is smooth, Eq. (1) is simplified, and takes the following form highly convenient for uses in conjunction with the temperature conductivity equation (see, e.g., Ref. [20]):

$$
\frac{\partial c}{\partial t}-D \Delta c=D_{T} c(1-c) \Delta T
$$

To arrive at Eq. (3) from Eq. (1), let us write $\nabla(c \nabla T)$ $=c \nabla^{2} T+\nabla T \nabla c$, and take into account that spatial variations of the concentration arise due to temperature gradients. At the steady state, the relationship between the temperature and the concentration gradients is determined by the so called Soret-Ludwig constant $S_{T}=D_{T} / D$ [23-25]: $\nabla c=$ $-c S_{T} \nabla T$. Typically, the quantity $c S_{T}$ is small, which means that the gradients of the concentration are much smaller compared to the gradients of the temperature. Thus $\nabla T \nabla c$ $\sim c S_{T}(\nabla T)^{2}$. Now let us note that $c S_{T}(\nabla T)^{2} \ll c \nabla^{2} T$, due both to the presence of the small parameter $c S_{T}$ and to the smallness related to $(\nabla T)^{2}$ : first of all, for any symmetric beam, $(\nabla T)^{2}$ vanishes on the axis of the beam, and, second, out of the beam, the term proportional to $(\nabla T)^{2}$ decays much faster than the term $\nabla^{2} T$. This justifies the transformation of Eq. (1) into Eq. (3). In this section, we will actually be considering a problem linearized with respect to small variations of the concentration, and will therefore have $c=c_{0}=$ const on the right-hand side of Eq. (1), that will directly convert it into Eq. (3).

The set of equations (2) and (3) can be simplified even further if we take into account the smallness of the so called Lewis number, the ratio of the mass diffusion constant, and the constant of the temperature conductivity; $D / \chi$ is typically of the order of $10^{-3}$ for liquids. This means that the temperature profile is established much faster than the spatial distribution of the concentration. Therefore, one can adiabatically eliminate the temperature from Eq. (3) by substituting

$$
\Delta T=-\frac{\sigma I}{\chi \rho C_{p}}
$$

in it. Thus we are left with the following equation:

$$
\frac{\partial c}{\partial t}=D \Delta c-D_{T} c(1-c) \frac{\sigma I}{\chi \rho C_{p}} .
$$

The most interesting properties of the physical system under consideration arise due to the dependence of the absorption constant $\sigma$ on the concentration $c$. This dependence is essentially important for systems containing strongly absorbing mobile centers (molecules or nanoparticles). Such a dependence provides feedback which can be both positive and

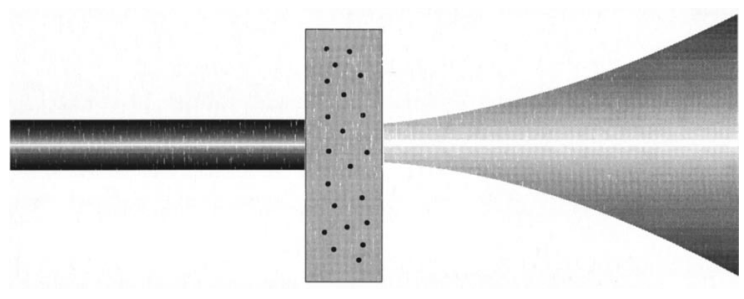

FIG. 1. The experimental scheme: a laser beam of a Gaussian profile of intensity incidents upon a suspension of absorbing particles (black dots). The divergence and the profile of the output beam is modified due to both the modulation of the particle concentration, the temperature of the medium and, consequently, the refractive index of the liquid.

negative, as determined by the sign of the thermal diffusion constant $D_{T}$. For a small concentration of the absorbers, we can simply accept

$$
\sigma=\alpha c
$$

where $\alpha$ is the absorption coefficient for the pure absorbent.

\section{REDISTRIBUTION OF ABSORBING PARTICLES IN A GAUSSIAN BEAM}

Consider a conventional scheme of a laser beam interacting with a liquid containing absorbing particles (Fig. 1). We will show here that the Soret effect plays a crucial role in determining the profile of the distribution of the absorbing particles and, thus, the profile of the temperature established in the medium. The escape of the particles from the region of maximal radiation intensity provides a negative feedback in many systems, limiting the heat release in the medium.

We will restrict ourselves to the two-dimensional (2D) problem, where the temperature gradients and the attenuation of the laser beam along the thickness of the cell are neglected. This assumption is very useful to reveal the qualitative features of the phenomena under discussion, to provide for explicit dependencies, and to allow for evaluations of experimental investigations. Taking into account the presence of the gradients along the thickness of the cell will modify the problem, but it will not change the qualitative features of the phenomena under discussion. The 2D problem has previously proven to be satisfactory in describing thermal nonlinear optical phenomena in absorbing materials.

For the steady state, Eq. (5), taking account of Eq. (6) and the expression for the intensity distribution in a Gaussian beam of the radius $w$,

$$
I(r)=I_{o} e^{-(r / w)^{2}},
$$

can be represented in the form

$$
\frac{1}{r} \frac{d}{d r}\left(r \frac{d c}{d r}\right)=\frac{\alpha S_{T} c^{2}(1-c) I_{o}}{\rho C_{p} \chi} e^{-(r / w)^{2}} .
$$

We will assume now that the homogeneous concentration $c_{0}$ of particles at the absence of the light beam is small, $c_{0}$ $\ll 1$, and that it undergoes small perturbation due to the lightinduced Soret effect, $c=c_{0}+c_{s}, c_{s} \ll c_{0}$. Then the perturba- 
tion of concentration can be determined from Eq. (8), linearized with respect to the intensity of the laser beam:

$$
\frac{1}{r} \frac{d}{d r^{\prime}}\left(r^{\prime} \frac{d c^{\prime}}{d r^{\prime}}\right)=\eta e^{-r^{2}}
$$

where

$$
\eta=S_{T} \frac{\sigma_{0} I_{o} w^{2}}{\rho C_{p} \chi}
$$

and $\sigma_{0}=\alpha c_{0}$. In Eq. (9), $r^{\prime}=r / w$ and $c^{\prime}=c_{s} / c_{0}$. In the following, we will omit the prime sign at $r$.

Integration of Eq. (9) yields

$$
\begin{aligned}
c^{\prime}(r) & =c^{\prime}(0)+\frac{\eta}{4}\left(1-e^{-r^{2}}\right) \ln \left(r^{2}\right)-\eta \int_{0}^{r} x \ln (x) e^{-x^{2}} d x \\
& =c^{\prime}(0)+\frac{\eta}{4} \ln \left(r^{2}\right)-\frac{\eta}{4} E_{i}\left(-r^{2}\right)+\frac{\eta \gamma}{4},
\end{aligned}
$$

where $c^{\prime}(0)$ is the concentration perturbations on the axis of the beam, $E_{i}\left(-r^{2}\right)$ is the exponential integral function, and $\gamma=0.577 \ldots$ is the Euler's number. The value of $c^{\prime}(0)$ is determined from the condition that the overall number of particles in a cylindrical cell containing the mixture is constant:

$$
\int_{0}^{R} c^{\prime}(r) r d r=0
$$

where $R$ is the size of the cell in units of the beam waist radius $w$. Finally, the expression for the concentration redistribution will be obtained as follows:

$$
c^{\prime}(r)=\frac{\eta}{4}\left[\ln \left(\frac{r}{R}\right)^{2}-E_{i}\left(-r^{2}\right)+E_{i}\left(-R^{2}\right)+1-\frac{1-e^{-R^{2}}}{R^{2}}\right] .
$$

The number of particles on the axis of the beam $r=0$ decreases according to

$$
c^{\prime}(0)=-\frac{\eta}{4}\left[\ln \left(R^{2}\right)+\frac{1-e^{-R^{2}}}{R^{2}}-E_{i}\left(-R^{2}\right)-1+\gamma\right] .
$$

Near the center of the beam, the radial dependence of the concentration perturbations is parabolic:

$$
c^{\prime}(r \sim 0) \approx c^{\prime}(0)+\frac{\eta}{4} r^{2} .
$$

Close to the boundary, the concentration is linearly increasing:

$$
c^{\prime}(r \sim R)=-\frac{\eta}{4}\left[-2\left(1-\frac{r}{R}\right)\left(1+e^{-R^{2}}\right)+1-\frac{1-e^{-R^{2}}}{R^{2}}\right] .
$$

At the boundary of the cell, $r=R$, the increase in the concentration of particles is

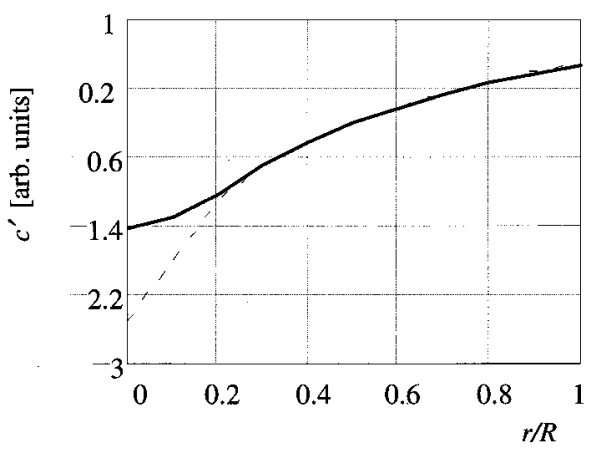

FIG. 2. The radial profile of the concentration perturbations for different sizes of the system: $R=5$ (solid line) and $R=15$ (dashed line).

$$
c^{\prime}(R)=\frac{\eta}{4}\left(1-\frac{1-e^{-R^{2}}}{R^{2}}\right)
$$

At the limit of a large cell size compared to the beam waist, $R \gg 1$, the above obtained formulas are reduced to

$$
\begin{gathered}
c^{\prime}(r) \approx \frac{\eta}{4}\left[\ln \left(\frac{r}{R}\right)^{2}-E_{i}\left(-r^{2}\right)+1-\frac{1}{R^{2}}\right], \\
c^{\prime}(0) \approx-\frac{\eta}{4}[2 \ln (R)-1+\gamma], \\
c^{\prime}(R) \approx \frac{\eta}{4}\left(1-\frac{1}{R^{2}}\right) .
\end{gathered}
$$

As evident from expressions (18)-(20), the concentration distribution depends on the size of the system $R$. This is a rather slow, logarithmic dependence in the case of large systems $R \gg 1$. This dependence is due to the confining role of the boundaries that do not let the particles escape to infinity.

In the case of small confinements, $R \ll 1$, we obtain

$$
\begin{gathered}
c^{\prime}(r)=\frac{\eta}{4}\left[\ln \left(r^{2}\right)-E_{i}\left(-r^{2}\right)+\gamma-\frac{1}{2} R^{2}\right] \\
=\frac{\eta}{4}\left[-\sum_{k=1}^{\infty} \frac{\left(-r^{2}\right)^{k}}{k \cdot k !}-\frac{1}{2} R^{2}\right], \\
c^{\prime}(0)=-\frac{\eta}{8} R^{2}, \\
c^{\prime}(R)=\frac{\eta}{8} R^{2} .
\end{gathered}
$$

Figure 2 demonstrates the radial distribution of the concentration perturbation for the two different values of the size of the system. Figure 3 presents the dependencies of the perturbations of the on-axis concentration and the concentration at the boundary as a function of the size of the system.

The absolute value of the variations in the concentration is determined by the parameter $\eta$. Noting that $\pi w^{2} I$ is the total power of the beam $P$, we can rewrite $\eta$ as 


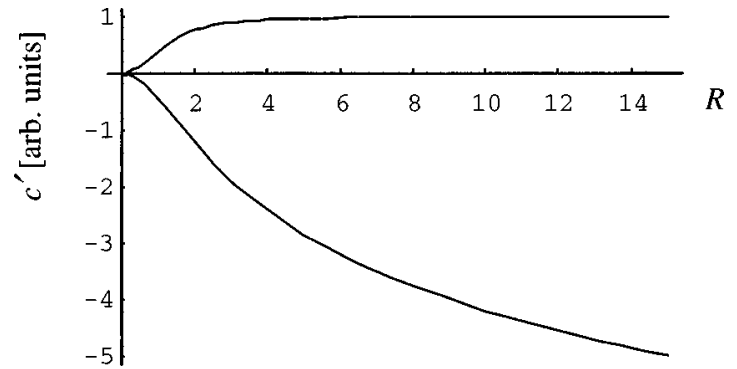

FIG. 3. The concentration perturbations on the axis of the Gaussian beam (decreasing curve) and at the boundary of the cell (increasing curve) as a function of the cell size.

$$
\eta=\frac{\sigma_{0} S_{T} P}{\pi \rho C_{p} \chi} .
$$

The magnitude of the effect is determined by the power of the laser radiation rather than by its intensity. This is explicitly shown by Eq. (19), where we see that the dependence on the beam size is logarithmic (recall that $R$ is the size of the system reduced to the beam waist radius $w$ ). Physically, the power dependence is a result of the circumstance that, at a fixed power, the smaller the beam waist radius, the larger the power density, but the smaller the gradients of the temperature and concentration.

Let us carry out a numerical estimation. Typically, for conditions of the experiment $[8,9], \sigma_{0} \sim 400 \mathrm{~cm}^{-1}, \rho C_{p}$ $\sim 1 \mathrm{~J} / \mathrm{cm}^{3} \mathrm{~K}$, and $\chi \sim 10^{-3} \mathrm{~cm}^{2} / \mathrm{s}$. The magnitude of $S_{T}$ has not yet been measured for magnetic fluids. As mentioned above, $S_{T}$ is very sensitive to a number of parameters characterizing the shape and size of the particles and their interactions, and there are no satisfactory theories to estimate the value of $S_{T}$ for liquid solutions. Thus, for particles of polystyrene of less than 100-nm size in dilute solutions in toluene, the Soret coefficient has been measured to be $S_{T}$ $=0.36 \mathrm{~K}^{-1}[26]$. For different sizes of polystyrene particles, similar values of $S_{T}$ were obtained in Ref. [27]. In a large number of situations, $S_{T}$ was found to be of the order of $10^{-3} \mathrm{~K}^{-1}[20,26,28]$. Let us assume here a rather small value for $S_{T}$ obtained in solutions of particles, $S_{T}$ $\sim 10^{-3} \mathrm{~K}^{-1}$. Then Eq. (24) yields $\eta \sim 1$ for a laser power as low as $10 \mathrm{~mW}$.

With account of the concentration profile (14), Eq. (4) can be directly integrated to yield the temperature profile established in the medium due to the absorption of a Gaussian beam. The integrals, however, are rather cumbersome, and we cannot present them explicitly through analytical functions.

\section{SORET FEEDBACK}

Let us proceed with a simple physical discussion to gain more insight into the qualitative features of the concentration distribution over the cell. That is, the absence of a mass flux in the equilibrium state relates the gradient of the concentration to the gradient of the temperature as follows:

$$
\frac{d c^{\prime}}{d r}=-S_{T} \frac{d T}{d r}
$$

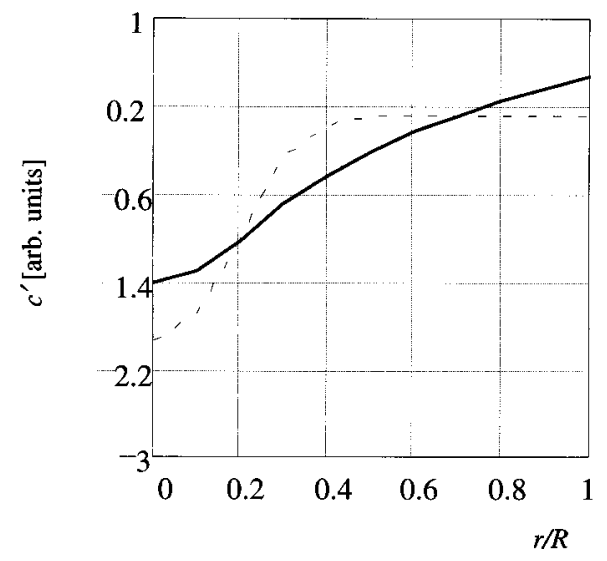

FIG. 4. The concentration profile obtained with the aid of the qualitative approach (dashed line). The solid line presents the plot of the correct solution [Eq. (14)] for comparison. Both curves are for $R=5$. The approximate solution assumes $\eta=1$.

The solution of Eq. (25) is

$$
T(r=0)-T(r)=\frac{c^{\prime}(r)-c^{\prime}(0)}{S_{T}} .
$$

Typically, the transverse size of the cells that contain the magnetic fluid is about $1 \mathrm{~cm}$, and the spot size of a focused laser beam on the cell is only tens of microns. Thus, the region that is heated by the beam is highly localized. Therefore, the change in the temperature at the boundary $r=R$ is negligible, and that boundary essentially maintains the room temperature. Thus we will assume a fixed temperature at the boundary $r=R$ of the cell, and we will rewrite Eq. (26) in the form

$$
T(r)-T(R)=\frac{c^{\prime}(R)-c^{\prime}(r)}{S_{T}} .
$$

On the other hand, the change in the temperature due to the absorption of the laser radiation can be estimated as

$$
T(r)-T(R)=\frac{\sigma_{0} I(r) \tau}{\rho C_{p}}\left[1+c^{\prime}(r)\right],
$$

where $\tau \sim w^{2} / \chi$ is the thermal relaxation time. Considering Eqs. (27) and (28) as a system of equations for $c^{\prime}(r)$ and $T(r)$, we arrive at the following solution for $c^{\prime}(r)$ :

$$
c^{\prime}(r)=-\frac{\eta e^{-r^{2}}-c^{\prime}(R)}{1+\eta e^{-r^{2}}} \approx-\frac{\eta e^{-r^{2}}}{1+\eta e^{-r^{2}}},
$$

where $c^{\prime}(R)$, determined by condition (13) to have a fixed number of particles in the volume, is considered to be negligibly small at the limit $R \gg 1$ :

$$
c^{\prime}(R) \approx \frac{\ln (1+\eta)}{R^{2}} \approx 0 .
$$

The spatial profile of $c^{\prime}(r)$ found from Eq. (29) is presented in Fig. 4, along with the plot of $c^{\prime}(r)$ according to the 


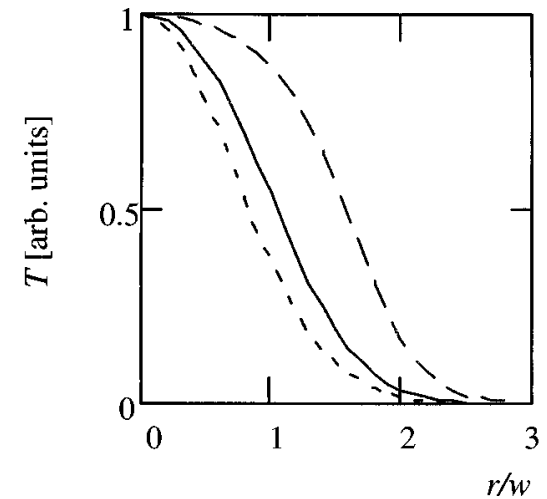

FIG. 5. The normalized temperature profile for different values of the parameter $\eta: \eta=10$ (dashed line), $\eta=1$ (solid line), and $\eta$ $\sim 0$ (short-dashed curve).

quantitative expression (14). Figure 4 demonstrates that the simple approach retains the main qualitative features of the process.

Equations (28) and (29), with account of $T(R) \sim$ $\exp \left(-R^{2}\right) \sim 0$ and $c^{\prime}(R) \rightarrow 0$, lead to the following expression for the change in the temperature:

$$
T(r)=\frac{1}{S_{T}} \frac{\eta e^{-r^{2}}}{1+\eta e^{-r^{2}}} .
$$

The spatial profiles of the temperature at different power levels are presented in Fig. 5. The most important consequence of Eq. (31) is that the increase in the temperature remains limited even for a high power of the incident radiation. The temperature on the axis of the beam is equal to

$$
T(r=0) \approx \frac{1}{S_{T}}
$$

at the limit $\eta \gg 1$. Thus the on-axis temperature is determined by the value of the Soret constant, and can serve as a method for its determination. Note that all details about the experimental parameters (beam power and waist size, size of the cell, and material parameters like temperature conductivity coefficient) do not appear in expression (32).

\section{EFFECT OF THE ELECTROSTRICTIVE FORCE}

While thermal diffusion is the main mechanism which causes motion and redistribution of strongly absorbing particles in the electromagnetic field of the light beam, the electrostriction forces resulting from the transverse modulation of beam intensity may still have an effect on the redistribution of particles. To evaluate their role, we have to include the electrostriction force into equations for the concentration

$$
\mathbf{F}=\frac{1}{2} \beta \nabla E^{2}=\frac{4 \pi \beta}{\bar{c} n} \nabla I,
$$

acting on a particle of polarizability $\beta[29,30]$. In Eq. (33), $n$ is the refractive index of the medium, and $\bar{c}$ is the speed of light in vacuum. Polarizability of a dielectric sphere of radius $a$ can be taken as

$$
\beta=\varepsilon_{h} \frac{\varepsilon_{p}-\varepsilon_{h}}{\varepsilon_{p}+2 \varepsilon_{h}} a^{3}
$$

Equation (3) for the concentration now can be written down in the form $[31,32]$

$$
\frac{\partial c}{\partial t}=D \Delta c+D_{T} c(1-c) \Delta T-\frac{D}{k_{B} T} \nabla c \mathbf{F} .
$$

In the framework of the approximations made above, the equation for the steady state distribution of the concentration assumes the form

$$
\Delta c+S_{T} c_{0} \Delta T-\frac{c_{0}}{I_{E}} \Delta I=0
$$

where we have introduced a notation

$$
I_{E}=\frac{\bar{c} n k_{B} T}{4 \pi \alpha} .
$$

Further, evaluating the solution of Eq. (36) as

$$
c^{\prime}=\frac{I}{I_{E}}-S_{T} T
$$

and relating the change in the temperature to the light intensity with the aid of expression (28), we obtain

$$
c^{\prime} \approx-\frac{I}{I_{S}}\left(1-\frac{I_{S}}{I_{E}}\right)
$$

where

$$
I_{S}=\frac{\rho C_{p} \chi}{S_{T} \sigma_{0} c_{0} w^{2}} .
$$

In obtaining Eq. (39), we assumed $T(R)=0$, and neglected the saturation effects.

Thus, in the case $\varepsilon_{p}>\varepsilon_{h}$, which takes place in solutions of magnetic particles, the electrostrictive forces drive the particles toward the higher intensity central region of the beam competing with the thermal diffusion, which forces particles out of the center of the beam. The relative forces of the two effects are determined by the ratio $I_{S} / I_{E}$. Let us first evaluate $\beta$. For magnetic particles of radius $a \sim 10^{-6} \mathrm{~cm}$ in kerosene, we have $\beta \sim 10^{-18} \mathrm{~cm}^{3}$, and, at room temperature, $I_{E} \sim 10^{7} \mathrm{~W} / \mathrm{cm}^{2}$. The magnitude of $I_{S}$ depends on the beam waist radius $w$. Assuming $w \sim 10 \mu \mathrm{m}, \sigma_{0}=400 \mathrm{~cm}^{-1}$, and $S_{T} \sim 10^{-3} \mathrm{~K}^{-1}$, we obtain $I_{S} \sim 310^{4} \mathrm{~W} / \mathrm{cm}^{2}$.

The strong dependence of the ratio $I_{S} / I_{E}$ on the size of the particles and on the beam waist radius makes it possible to obtain comparable values of $I_{S}$ and $I_{E}$ by minor modifications of the experimental parameters. Actually, we do not precisely know the values of $a$ and $S_{T}$ for the case of the magnetic particles explored in Refs. [8,9], while the beam width in those experiments was even smaller than accepted in estimations made above. 


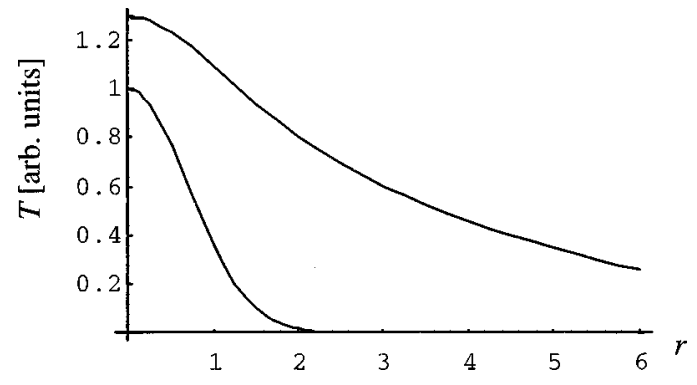

FIG. 6. The temperature profile calculated with the aid of the correct quantitative expressions (upper curve) and its Gaussian approximation (lower curve): the boundary condition corresponds to the fixed temperature $(T=0)$ at $R=10$.

\section{FUNDAMENTAL DISBALANCE OF ELECTROSTRICTIVE AND THERMAL COUNTERDIFFUSIONS}

Evaluation of the temperature with the aid of expression (28) oversimplifies the physical pictures of the processes under discussion, and masks the following interesting effect. That is, the correct solution of the temperature conductivity equation for the Gaussian beam, first obtained in Ref. [33], can be presented in the following way to facilitate the calculations:

$$
T(r)=T_{0} f(r, R),
$$

where $T_{0}=\sigma I_{0} w^{2} / \chi \rho C_{p}=\sigma P / \pi \chi \rho C_{p}=I_{0} / S_{T} I_{S}$ coincides with the "Gaussian approximation' for the temperature rise estimated with the aid of expression (28), and

$$
f(r, R)=\frac{1}{4}\left\{E_{i}\left(-r^{2}\right)-\ln \left(r^{2}\right)-\left[E_{i}\left(-R^{2}\right)-\ln \left(R^{2}\right)\right]\right\} .
$$

Figure 6 presents the correct profile of the temperature found out from Eqs. (41) and (42), as well as the Gaussian profile. As one can see, the actual profile of the temperature is remarkably smoother than its Gaussian approximation as soon as the size of the system is larger than the beam waist radius. To fit the actual complex temperature profile with a Gaussian function, the effective width of the fitting Gaussian would have to be remarkably larger. However, the Gaussian approximation describes fairly well the maximal rise in the temperature obtained on the axis of the beam acting on a usual (linear) absorbing medium (Fig. 7).

Thus the competition between the electrostrictive and thermal-diffusion processes is not reduced just to quantita-

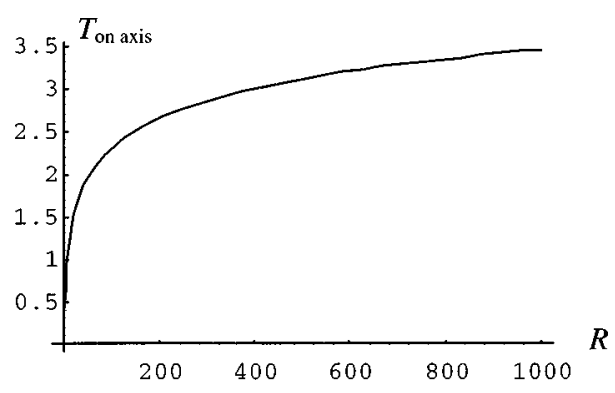

FIG. 7. The ratio of the temperature on the axis of the beam to the "Gaussian" maximal temperature as a function of the size of the system.

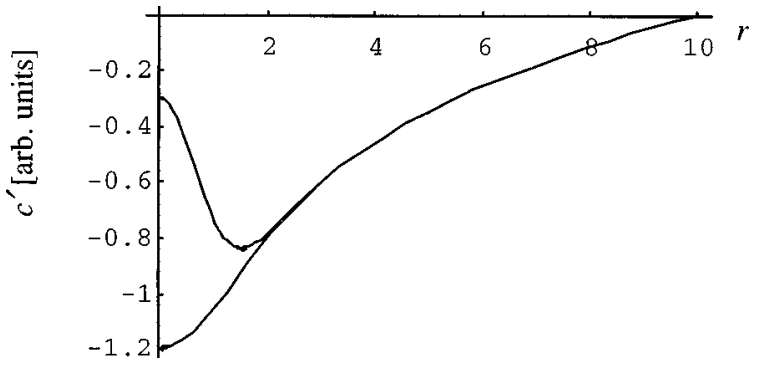

FIG. 8. The concentration profile taking account of the competing action of the electrostriction and the thermal diffusion $(R$ $=10$ ). The nonmonotonous behavior of the curve reveals the fundamental misbalance of counterdiffusions for equivalently strong forces. The monotonous curve corresponds to the electrostrictive diffusion that is an order of magnitude smaller.

tive changes in the magnitude of the concentration redistribution, but it influences the profile of the concentration distribution as a whole. According to Eqs. (38) and (41), the resultant profile can be written down as

$$
c^{\prime} \approx-\frac{I_{0}}{I_{S}}\left[f(r, R)-\frac{I_{S}}{I_{E}} e^{-r^{2}}\right] .
$$

Figure 8 shows the obtained concentration distribution for the particular values of the ratio $I_{S} / I_{E}$. In the case of comparably strong electrostriction and thermal-diffusion effects, the concentration first decreases with increasing distance from the axis of the beam, and then increases again, since the action of the striction forces becomes negligible at the periphery of the beam.

We may try to evaluate the effect of the feedback by assuming in Eq. (41) that $\sigma=\sigma_{0}(1+c)$, and solving the system of equations (38) and (41). Then, expression (43) becomes simply multiplied by the factor $\left(1+I_{0} f(r, R) / I_{S}\right)^{-1}$, and the effect of the spatial disbalance between the electrostriction and the thermal diffusion on temperature is described by the expression

$$
T(r) \approx \frac{\left(I_{0} / I_{s}\right) f(r)}{1+\left(I_{0} / I_{s}\right) f(r)}\left[1+\frac{I_{0}}{I_{E}} e^{-r^{2}}\right] .
$$

Thus, though the concentration may accept a nonmonotone distribution in space, the temperature increase is always maximal at the center of the beam, even in circumstances of a strong feedback.

\section{PHASE MODULATION OF THE LASER BEAM AND RELATED PATTERNS}

Variations in concentration of the particles and the temperature of the liquid yields variations of the refractive index of the material:

$$
\delta n=\left(\frac{\partial n}{\partial T}\right)_{c} T+\left(\frac{\partial n}{\partial c}\right)_{T} c
$$

As we are concerned with the results of the experiments $[8,9]$, let us carry out evaluations for the kerosene-based magnetic fluid. The temperature dependence of the refractive index of light petroleum oils has proven to be very large: 
$\partial n / \partial T \sim-10^{-3} \mathrm{~K}^{-1}$ [34]. The concentration dependence of the refractive index of mixtures can be evaluated with the aid of the Lorentz-Lorentz formula [30,35]. We can find the following correction to the refractive index of the host liquid:

$$
\delta n=\frac{3 n_{h}}{2} \frac{\varepsilon_{p}-\varepsilon_{h}}{2 \varepsilon_{h}+\varepsilon_{p}} c,
$$

where $\varepsilon_{h}=n_{h}^{2}$ and $\varepsilon_{p}=n_{p}^{2}$ are the dielectric susceptibilities of the mixture and the host medium, respectively. In Eq. (46), $\varepsilon_{p}$ is the real part of the dielectric susceptibility of the magnetic particles, which is known to be $\varepsilon_{p}=7.75$ for $\mathrm{Fe}_{3} \mathrm{O}_{4}$ at a 630-nm wavelength of the optical radiation [36]. Taking into account also the value of the refractive index of the kerosene, $n_{h} \approx 1.5$, one can determine $\partial n / \partial c \approx 1.2$ from Eq. (46). Thus both effects, escape of particles from the region of high temperature and the temperature increase by itself, result in a decrease of the effective refractive index of the mixture. From that, in the case of magnetic particles, we may expect a major contribution to the changes in the refractive index from the concentration redistribution.

As is well known, bell-shaped (particularly Gaussian) phase modulation leads to a characteristic pattern of annular rings. This pattern is especially striking in the case of orientational optical nonlinearity of liquid crystals, where, due to the giant values of the optical nonlinearity, the phase modulation and the related number of rings are enormously large [37]. The number of these rings carries information about the maximal phase shift achieved on the axis of the beam.

To demonstrate several typical pictures and the saturation effect induced by the Soret feedback, let us use the simplified expression (32) to obtain the profile of the self-phase modulation of the laser beam due to the interaction with the solution. Then one can present the nonlinear phase shift in the following way:

$$
\Phi=\Phi_{0} f(r),
$$

where

$$
\Phi_{0}=-\frac{2 \pi L}{\lambda} \frac{\eta}{1+\eta}\left[\left(\frac{\partial n}{\partial c}\right)_{T}-\frac{1}{S_{T}}\left(\frac{\partial n}{\partial T}\right)_{c}\right]
$$

is the phase shift on the axis of the beam, and

$$
f(r)=\frac{(1+\eta) e^{-r^{2}}}{1+\eta e^{-r^{2}}}
$$

determines the profile of the transverse self-phase modulation. Figure 9 shows the result of theoretical modeling of the patterns given rise at the far field zone by a beam with the phase profile (49). Figure 10 shows the distribution of the light intensity along the diameter of the pattern for several values of $\eta$. We see that increasing the heat insertion into the liquid (increasing light intensity, absorption of the fluid, and Soret constant) modifies the ring pattern by redistributing the intensity over the rings. This pattern, thus, carries information about the spatial distribution of the particles and the temperature. As shown in Ref. [38], the complex profile of the self-phase modulations can lead to diffraction catastrophes during propagation of the beam in free space.

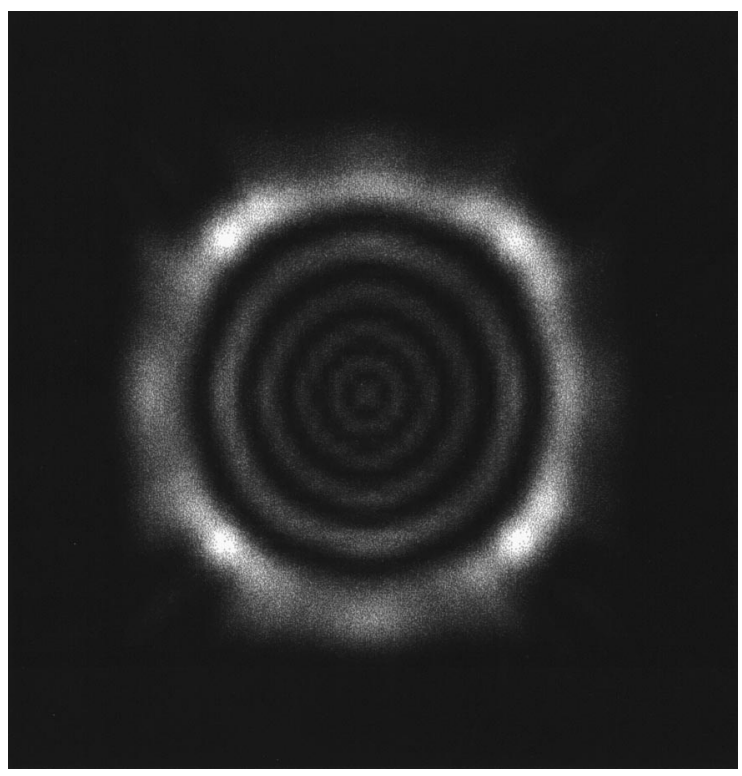

FIG. 9. Modeling of the typical far-field pattern of the self-phase modulation of the laser beam: $\Phi_{0}=12 \pi$ and $\eta=0$.

\section{OPTICAL TRANSMITTANCE OF "SORET CELLS"}

Redistribution of absorbing particles in the medium due to the Soret effect modifies the transmittance of the cell. Let us consider a medium, the absorption constant of which is a function of radial coordinate

$$
\sigma(r)=\sigma_{0}\left[1+c^{\prime}(r)\right] .
$$

Then the intensity of radiation in the medium will attenuate as

$$
I(r, z)=I_{0} e^{-(r / a)^{2}-\sigma(r) z} .
$$

Making use of expression (29) for the modification of the particle distribution, we can find the following expression for the power of radiation when crossing a distance $z$ in the medium:

$$
\begin{aligned}
P(z)= & 2 \pi \int_{0}^{\infty} I(r) r d r \\
= & P_{0} \frac{1}{\eta}\left\{\sigma z\left[E_{i}\left(-\frac{\sigma z}{1+\eta}\right)-E_{i}(-\sigma z)\right]\right. \\
& \left.+\eta e^{-\sigma z /(1+\eta)}-e^{-\sigma z}\left(1-e^{\sigma z \eta /(1+\eta)}\right)\right\},
\end{aligned}
$$

where $P_{0}$ is the total power of the incident radiation. In the limiting case of the small Soret effect, Eq. (52) is reduced to the usual Beer's law. The plot of $P(z)$ is presented in Fig. 11. As we see, the transmittance of the system can be remarkably affected due to the redistribution of particles. In the present example, where $\eta=3$, the transmittance is almost five times larger at $\sigma z=3$ due to the escape of particles from the region of maximal intensity than we would have for the same system in its homogeneous state. Thus less heat is being released in the medium and, therefore, less increase in the temperature is achieved compared to homogeneous mixture. Fitting the thickness dependence curve of the system's 

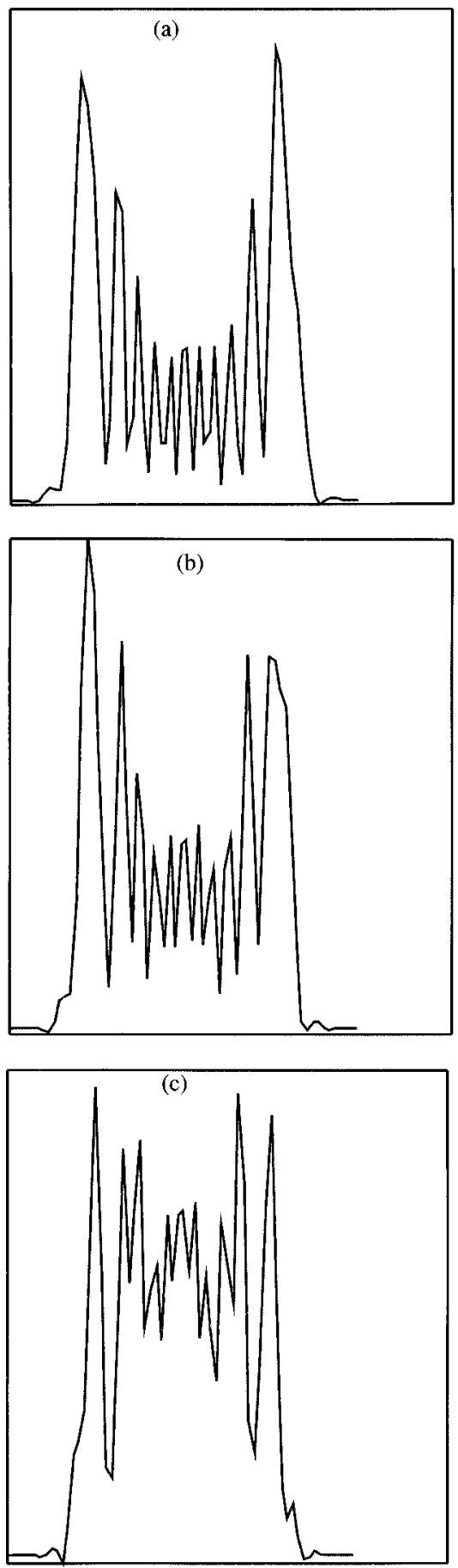

FIG. 10. The effect of the strength of the Soret feedback on the profile of the far-field patterns presented in Fig. 9: $\Phi_{0}=12 \pi$ : (a) $\eta=0$; (b) $\eta=0.3$; (c) $\eta=1$. Some asymmetry is caused by the numerical noise.

transmission will allow a determination of the Soret constant provided all the other parameters of the system are known.

\section{CONCLUSIONS}

There are several interesting processes that still have to be studied. Among them, the instabilities and dynamic phenomena inherent to nonlinear systems with feedback. One can distinguish two physical situations that may exhibit different

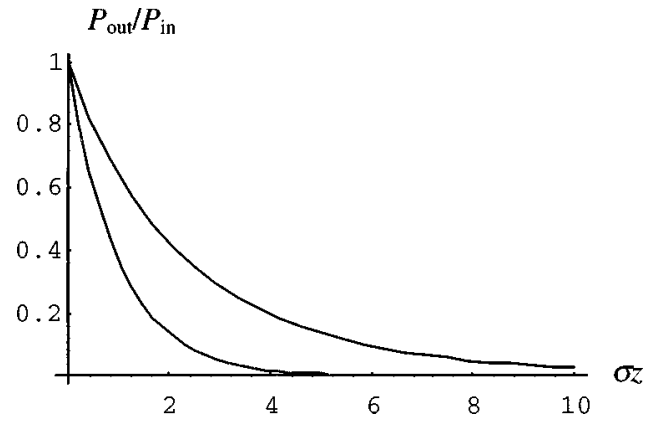

FIG. 11. The total power $P$ transmitted through the cell as a function of $\sigma z$ upper curve, with account of transverse redistribution of particles; lower curve, exponential absorption for homogeneous distribution of particles.

types of instabilities based upon the sign of the Soret constant. In the case $S_{T}<0$, the local increase of the temperature attracts the absorbing particles into the warmer region. This yields increased absorption, which further increases the temperature. Such a positive feedback may lead to an avalanche jump in the particle concentration and bistability.

Since strong temperature gradients are involved in the problem, it is important to be convinced that convective instabilities will not interfere with experimental studies of the phenomena under consideration. The presence of the Soret effect may have a remarkable influence on the value of the Rayleigh number that initiates the convective instabilities. Indeed, the density gradient is the result of particle redistribution in space due to thermal diffusion, as well as due to the thermal expansion of the liquid. Therefore, a positive Soret effect may destabilize the fluid, making the particles migrate from the bottom hot boundary toward the upper cold boundary of the cell. This phenomenon was studied in a number of papers [39-43]. The results of these studies (see, for example, the numerical evaluations obtained in Ref. [41]) indicate, however, that the threshold Rayleigh number stays large $(R \gg 1)$ for any reasonable value of the Soret constant, the thickness of the cell, and the gradient of temperature.

Now let us note that the magnetic fluids strongly absorb light even for only a few percent of particle concentration. In the experiments that initiated our present work, and that are cited in this paper, the absorption constant was about $\sigma$ $=500 \mathrm{~cm}^{-1}$. Consequently, the thickness of the layer of the magnetic colloid has to be rather small, typically, 30-100 $\mu \mathrm{m}$, to allow for the laser beam to travel through the cell. No convective instability is realistic in such circumstances: the Rayleigh number is rather small $(R \ll 1)$, even if the temperature gradient is as huge as $10^{5} \mathrm{~K} / \mathrm{cm}$.

Convective motions can be induced in the liquid also due to transverse thermal gradients. As discussed in Ref. [44], a steady circulation can be generated for $R<10^{3}$, and the characteristics of this circulation depend essentially on the magnitude of the thickness of the magnetic fluid layer. Even if such a circulation will set in the cell, its velocity will apparently be negligibly small for a cell thickness of only several tens of microns.

As discussed in the present paper, the Soret effect may lead to a negative feedback: the absorbing particles escape 
from the hot regions to the cooler parts, thus decreasing the absorption. In an essentially nonlinear regime we may expect temporal instabilities and oscillations: escape of particles from the axis of the beam (warmer region) decreases the absorption; the temperature drops and the particles are driven back to the axis of the beam, and the cycle is repeated.

The basic equation (5) suggests that there might also be a spatial instability for a plane wave, with the spatial scale of the instability determined by the intensity of radiation. Physically, fluctuational modulations in the density of particles result in modulations in the temperature, which destroys the homogeneous spatial modulation of the particles due to the Soret effect.

The role of the Soret effect in nonlinear optics has actually not been discussed so far in full details. New interesting possibilities may arise for dynamic holography and optical storage. Thus the separation of charged molecules of different origins in the interference pattern of the light beams, and the associated space charge field, may underlie new mechanisms of photorefractivity, particularly in liquid crystals, where even relatively small electric fields can lead to a remarkable reorientation of the optical axis of the liquid crystal.
We have discussed thermal interaction of a laser radiation with liquids containing mobile absorbing particles or molecules. We have found a spatial distribution of particles and temperature in a Gaussian laser beam, and have shown that feedback due to the Soret effect limits the temperature increase in the medium. A nonlinear optical method to determine the Soret constant has been suggested which may combine the accuracy of optical methods with the simplicity of its realization. The obtained results allowed an interpretation of certain observations in magnetic fluids. We have outlined practical and fundamentally interesting problems for future investigations.

\section{ACKNOWLEDGMENTS}

The authors are grateful to T. Du and S. R. Nersisyan for valuable discussions. The research of N.V.T. and W.L. was supported by the NSF Young Investigator Award of Weili Luo. The research of N.V.T. was also partially supported by Air Force Grant No. F49620-95-1-0520.
[1] R. E. Rosensweig, Ferrohydrodynamics (Cambridge University Press, Cambridge, 1985).

[2] M. I. Shilomis, Sov. Phys. Usp. 17, 153 (1974).

[3] M. Kreuzer, T. Tschudi, W. H. de Jeu, and R. Eidenschink, Appl. Phys. Lett. 62, 1712 (1993).

[4] R. S. Akopyan, N. V. Tabiryan, and T. Tschudi, Phys. Rev. E 49, 3143 (1994).

[5] A. J. Palmer, Opt. Lett. 5, 54 (1980).

[6] P. W. Smith, P. J. Maloney, and A. Ashkin, Opt. Lett. 7, 347 (1982).

[7] E. Freysz, M. Afifi, and A. Ducasse, J. Phys. (France) Lett. 46, L-181 (1985).

[8] T. Du, S. Yuan, and Weili Luo, Appl. Phys. Lett. 65, 1844 (1994).

[9] T. Du and Weili Luo, Mod. Phys. Lett. B 9, 1643 (1995).

[10] Yu. I. Dikansky and V. P. Shatsky, J. Magn. Magn. Mater. 85, 82 (1990).

[11] Yu. I. Dikansky and A. O. Tsebers, Magn. Gidrodin. 2, 47 (1990).

[12] J. C. Bacri, A. Cebers, A. Bourdon, G. Demouchy, B. M. Heegaard, and R. Perzynski, Phys. Rev. Lett. 74, 5032 (1995).

[13] I. Janossy, Phys. Rev. E 49, 2957 (1994).

[14] E. V. Rudenko and A. V. Sukhov, Zh. Eksp. Teor. Fiz̀. 105, 1621 (1994) [ Sov. Phys. JETP 78, 875 (1994)].

[15] I. C. Khoo, H. Li, and Y. Liang, Opt. Lett. 19, 1723 (1994).

[16] K. Thyagarajan and P. Lallemand, Opt. Commun. 26, 54 (1978).

[17] H. J. Eichler, P. Guenter, and D. W. Pohl, Laser-Induced Dynamic Gratings (Springer-Verlag, Berlin, 1986).

[18] D. W. Pohl, Phys. Lett. A 77, 53 (1980).

[19] F. Bloisi, L. Vicari, P. Cavaliere, S. Martellucci, J. Quartieri, P. Mormile, and G. Pierattini, Appl. Phys. B: Photophys. Laser Chem. 44, 103 (1987).
[20] W. Koehler, J. Chem. Phys. 98, 660 (1993).

[21] B. Jean-Jean, E. Freysz, A. Ponton, A. Ducasse, and B. Pouligny, Phys. Rev. A 39, 5268 (1989).

[22] J. P. Delville, C. Lalaude, E. Freysz, and A. Ducasse, Phys. Rev. E 49, 4145 (1993).

[23] K. E. Grew and T. L. Ibbs, Thermal Diffusion in Gases (Cambridge University Press, Cambridge, 1952).

[24] S. R. de Groot and P. Mazur, Non-Equilibrium Thermodynamics (North-Holland, Amsterdam, 1962).

[25] L. D. Landau and E. M. Lifshitz, Fluid Mechanics (Pergamon, Oxford, 1987).

[26] W. Koehler and P. Rossmanith, J. Phys. Chem. 99, 5838 (1995).

[27] P. M. Shiundu, G. Liu, and J. Calvin Giddings, Anal. Chem. 67, 2705 (1995).

[28] T. R. Griffiths and N. J. Phillips, J. Electrochem., Soc. 138, 3575 (1991).

[29] J. P. Gordon, Phys. Rev. A 8, 14 (1973).

[30] L. D. Landau, E. M. Lifshitz, and L. P. Pitaevskii, Electrodynamics of Continuous Media (Pergamon, Oxford, 1984).

[31] N. Bloembergen, W. H. Lowdermilk, M. Matsuoka, and C. S. Wang, Phys. Rev. A 3, 404 (1971).

[32] D. Rogovin and S. O. Sari, Phys. Rev. A 31, 2375 (1985).

[33] J. P. Gordon, R. C. C. Leite, R. S. Moore, S. P. S. Porto, and J. R. Whinnery, J. Appl. Phys. 36, 3 (1965).

[34] V. A. Kalichevsky and K. A. Kobe, Petroleum Refining with Chemicals (Elsevier, Amsterdam, 1956).

[35] N. Rebbouh and J. R. Lalanne, J. Chem. Phys. 90, 1175 (1989).

[36] H. W. Davies and J. P. Llewellyn, J. Phys. D 13, 2327 (1980).

[37] N. V. Tabiryan, B. Ya. Zel'dovich, and A. V. Sukhov, The Orientational Optical Nonlinearity of Liquid Crystals, special 
issue of Mol. Cryst. Liquid Cryst. 136, 1 (1986).

[38] N. V. Tabiryan, B. Ya. Zel'dovich, M. Kreuzer, T. Vogeler, and T. Tschudi, J. Opt. Soc. Am. 13, 1426 (1996).

[39] M. G. Velarde and R. S. Schechter, Chem. Phys. Lett. 12, 312 (1971)

[40] D. T. J. Hurle and E. Jakeman, J. Fluid Mech. 47, 667 (1971).
[41] J. C. Legros, J. K. Platten, and P. G. Poty, Phys. Fluids 15, 1383 (1972).

[42] S. W. Joo, J. Fluid Mech. 293, 127 (1995).

[43] G. Zimmermann, U. Mueller, and S. H. Davis, J. Fluid Mech. 238, 657 (1992).

[44] J. W. Elder, J. Fluid Mech. 23, 77 (1965). 